\title{
Peer support for people with severe mental illness versus usual care: study protocol for a pragmatic multicentre randomised controlled trial (UPSIDES- $\mathrm{RCT}$ )
}

Galia Sharon Moran ( $\nabla$ galia.moran@gmail.com )

Ben-Gurion University of the Negev https://orcid.org/0000-0001-9718-1773

Jasmine Kalha

Indian Law Society

Annabel Mueller-Stierlin

Ulm University

Reinhold Kilian

Ulm University

Silvia Krumm

Ulm University

Mike Slade

University of Nottingham

Ashleigh Charles

University of Nottingham

Candelaria Mahlke

University Medical Centre Hamburg-Eppendorf

Rebecca Rebecca Nixdorf

University Medical Centre Hamburg-Eppendorf

David Basangwa

Butabika National Referral Hospital

Juliet Nakku

Butabika National Referral Hospital

Richard Mpango

Butabika National Referral Hospital

\section{Grace Ryan}

London School of Hygiene and Tropical Medicine

Donat Shamba

Ifakara Health Institute

Mary Ramesh

Ifakara Health Institute 
Fileuka Ngakongwa

Ifakara Health Institute

Alina Grayzman

Ben-Gurion University of the Negev

Soumitra Pathare

Indian Law Society

Benjamin Mayer

Ulm University

Bernd Puschner

Universitat Ulm

\section{Study protocol}

Keywords: Peer support, Severe Mental IIIness, Randomized Controlled Trial, Process Evaluation, CostEffectiveness Analysis, Implementation Science, Global Mental Health

Posted Date: January 7th, 2020

DOI: https://doi.org/10.21203/rs.2.20188/v1

License: (c) (i) This work is licensed under a Creative Commons Attribution 4.0 International License. Read Full License

Version of Record: A version of this preprint was published at Trials on May 1st, 2020. See the published version at https://doi.org/10.1186/s13063-020-4177-7. 


\section{Abstract}

Background: Peer support is an established intervention involving a person in recovery from mental illness being engaged to offering support to others with mental illness. Peers are an under-used resource in global mental health. Building upon comprehensive formative research, this study will rigorously evaluate the impact of peer support at the levels of service users (psychosocial and clinical outcomes), peer support workers (work role, empowerment), services (cost-effectiveness, return on investment), and implementation (adoption, sustainability, organisational change).

Methods: UPSIDES-RCT is a pragmatic parallel-group multi-centre randomised controlled trial assessing the effectiveness of UPSIDES at four measurement points over one year (baseline, 4-, 8-, and 12-month follow-up), and embedded process evaluation and cost-effectiveness analysis. Research will take place in a range of high-, middle- and low-income countries (Germany, United Kingdom, Israel, Uganda, Tanzania, India). The primary outcome is social inclusion of service users with severe mental illness ( $N=558 ; N=93$ per site) at 8-month follow-up, measured with the Social Inclusion Scale. Secondary outcomes include empowerment (Empowerment Scale), hope (HOPE scale), recovery (Stages of Recovery), and health and social functioning (Health of the Nations Outcome Scales). Mixed-methods process evaluation will investigate mediators and moderators of effect, and implementation experiences of four UPSIDES stakeholder groups (service users, peer support workers, mental health workers, and policy makers). A cost-effectiveness analysis examining cost-utility and health budget impact will estimate the value for money of UPSIDES peer support.

Discussion: By implementing and evaluating a manualized peer support intervention for people with severe mental illness across low-, middle-, and high-income countries, this study will contribute to harmonising core elements of peer support across different cultural and organisational dimensions. The UPSIDES-RCT will explore the essential components necessary to create a peer support model in mental health care, while providing the evidence required to sustain and eventually scale-up the intervention. Performance of mental health services will be maximised by actively involving and empowering service users, generating system changes towards user-centeredness, recovery orientation, community participation, and realising mental health as a human right.

Trail registration: ISRCTN, ISRCTN26008944. Registered 30 October 2019, http://www.isrctn.com/ISRCTN26008944.

\section{Background}

Peer support is part of a broader recovery agenda in mental health that places emphasis on user-centred outcomes such as social inclusion and empowerment [1]. Peer support is an established intervention whereby a person in recovery from mental illness is engaged to offer support to others with mental illnesses [2]. Peer support workers (PSWs) support their own recovery and the recovery of others by drawing on their lived experiences, employing positive self-disclosure, expanding social networks, and 
promoting hope, empowerment and self-efficacy. Around the world, diverse PSW roles have been developed and formalised: peer companions, peer advocates, consumer case managers, peer specialists, peer counsellors and more [3]. PSWs providers offer a wide range of services, which may include social support, management of symptoms, counselling, outreach, coaching and advocacy [4]. Peer support can also be provided in different settings - as an alternative to, an independent service within, or an integral part of professional care [5].

Findings from meta-analyses synthesizing a large number of controlled studies indicate that PSWs are able to achieve outcomes comparable to professionally trained staff, and might therefore represent costeffective additions to task-sharing models in low- and middle-income countries (LMICs) as well as in high-income countries (HICs) [6, 7]. Qualitative and quantitative studies have also shown that peer support has a positive impact on recovery-related outcomes that may not be possible to achieve using exclusively clinical interventions $[8,9]$. However, there are significant gaps in the evidence base for peer support in LMICs [10] and in non-Anglophone countries [11], with most reviews about peer support only identifying studies from HICs, primarily English-speaking countries [12].

The UPSIDES consortium

The project Using Peer Support In Developing Empowering Mental Health Services (UPSIDES) was designed to investigate the effectiveness and implementation of peer support in a range of high-, middleand low-income countries, in order to generate evidence on a scalable model of recovery-oriented mental health care that may be transferable to similar settings. UPSIDES is a research consortium involving eight collaborating institutions across six countries: Ulm University, Germany; University of Nottingham, UK; University Hospital Hamburg-Eppendorf, Germany; Butabika National Referral Hospital, Kampala, Uganda; London School of Hygiene and Tropical Medicine, UK; Ifakara Health Institute, Dar es Salaam, Tanzania; Ben-Gurion University of the Negev, Beer Sheva, Israel; and Centre for Mental Health Law and Policy, Pune, India (see www.upsides.org for further information).

UPSIDES takes place over a five-year period (2018-2022) and is divided into two phases, with different objectives and methods. In phase 1, which lasts two years, implementation stage of peer support across study sites will be assessed and a culturally appropriate peer support intervention will be developed [13]. This paper focuses on the three years of phase 2 , when the finalised peer support intervention will be implemented and evaluated at the levels of service users (psychosocial and clinical outcomes), peer support workers (empowerment, professional development) services (cost-effectiveness, return on investment), and implementation (adoption, sustainability, organisational change).

Framework of the current study

UPSIDES' conceptual background is the model of change processes in mental health peer support developed by Gillard et al [14] (Fig. 1). The model, based on a large multisite qualitative study, provides a measurable set of outcomes expected to change in response to processes of peer support. These address active ingredients of peer support and relate to a host of process outcomes of peer workers including 
hope, social functioning and increased engagement with services, and downstream impacts refer to recovery, well-being, and service use.

UPSIDES research is also guided by the Consolidated Framework For Implementation Research (CFIR [15], Fig. 2) which was created to promote implementation theory development, helping to standardise investigation into and verification into what works, where, and why across multiple contexts. CFIR covers five major domains that can influence effective implementation: (i) intervention, (ii-iii) outer and inner setting, (iv) individuals involved, and (v) process.

Building on results of formative research described elsewhere $[13,16]$, UPSIDES peer support will be implemented across sites in line with an implementation manual, taking into account differences across study sites. The development of the manuals as well as their implementation and the overall study design will take into consideration the five CFIR domains by, for example: incorporating adaptations to local contexts; planning for organizational readiness; paying attention to the selection and involvement of relevant stakeholders; and allowing for a staged approach to continuously modify the intervention as needed.

Objectives and research question

The aim of UPSIDES-RCT is to explore the implementation and effectiveness of peer support delivered in a range of high-, middle- and low-income country contexts. The main objectives are:

1. To evaluate the outcomes of delivering peer support, for service users, peer support workers and organisations, through a multi-centre pragmatic parallel-group randomised controlled trial and additional qualitative methods.

2. To assess the value for money of peer support for persons with severe mental illness, by carrying out a cost-effectiveness analysis.

3. To evaluate the process of implementing the UPSIDES peer support intervention, with special attention to differences in context across the study sites, using both quantitative and qualitative methods with PSWs, service users, mental health workers and wider stakeholders.

\section{Methods/design}

This protocol covers the UPSIDES pragmatic parallel-group multi-centre randomised controlled trial, costeffectiveness analysis, and process evaluation, described further below. This study protocol adheres to the SPIRIT statement [17].

\section{Setting}

Six of the eight UPSIDES collaborating institutions will host study sites:

1. Ulm, Germany: Catchment area of Ulm University's Department of Psychiatry and Psychotherapy II at institutions which provide UPSIDES peer support. 
2. Hamburg, Germany: University Medical Centre Hamburg-Eppendorf and community services all over Hamburg, which provide UPSIDES peer support. Peer support has been implemented in Hamburg since 2007.

3. Kampala, Uganda: The intervention will be provided at Butabika Hospital which is the main institution providing peer support in Uganda.

4. Dar es Salaam, Tanzania: The intervention will be implemented at Muhimbili National Hospital at the Department of Psychiatry and Mental Health.

5. Beer Sheva, Israel: The intervention will be implemented at institutions in Israel which provide UPSIDES peer support.

6. Pune, India: The intervention will be implemented at Hospital for Mental Health in Ahmedabad, Gujarat, which is a public mental health facility and has been implementing peer support since April 2015.

Randomised controlled trial

UPSIDES-RCT is a pragmatic parallel-group randomised controlled multicentre trial including a waiting list, with four measurement points ( $t_{0}=$ baseline; $t_{1}=4$ months; $t_{2}=8$ months; $t_{3}=12$ months $)$.

\section{Participants}

Inclusion criteria: Adult age (18-60 years) at intake; mental disorder of any kind as main diagnosis established by case notes, staff communication or self-label; presence of severe mental illness (Threshold Assessment Grid [18] $\geq 5$ points and illness duration $\geq 2$ years); sufficient command of the host country's language; capable of giving informed consent.

Exclusion criteria: Main diagnosis of learning disability, dementia, substance disorder or organic brain disorder; cognitive impairment severe enough to make it impossible to give informed consent or complete study measures.

Processes, intervention and comparison

Intervention

All participants will receive treatment as usual (TAU). Participants allocated to the intervention group will additionally receive the UPSIDES peer support, which is a direct service that is delivered by a person who has personal experience of mental health problems to a person or a group of persons with a serious mental illness. UPSIDES peer support workers (PSWs) will be persons of adult age, having experienced mental ill health and having progressed along in their recovery as indicated by having been stable or out of hospital for at least three months. Peer support workers will have progressed along in their recovery beyond controlling symptoms, to a focus on self-definition, growth and participation in the community. UPSIDES PSWs will be using these personal experiences, along with UPSIDES training and supervision, to facilitate, guide, and mentor another person's recovery journey [19]. Social support and recovery role 
modelling are central to UPSIDES peer support, while other elements may vary across sites depending on need and feasibility, e.g. management, counselling, outreach, coaching and advocacy. The intervention has been developed by all UPSIDES partners and the training manualized [13], based on and adapting existing programs: ImROC training (UK [20]), Ex-IN curriculum (Germany [21]), Brain Gain program (Uganda [22], QualityRights (WHO [23], India [24]), Healthy Options project (Tanzania [25]), Peer2peer (Europe [26]), and Yozma Derech-Halev consumer-provider training (Israel [27]).

The training has been manualized [28], with a complementary workbook addressing underlying values and principles, specific skill training and preparing PSWs for tasks around recovery planning. The UPSIDES peer support training consists of 12 core modules: (1) understanding recovery; (2) own recovery journey; (3) peer support; (4) communication; (5) recovery planning; (6) activating resources; (7) community and trialogue; (8) problem solving, (9) group facilitating; (10) peer role description; (11) work preparation; and (12) network. Adaptations are trained in additional modules which take into account context (social and environmental situation, resources in mental health care) and site-specific topics (e.g. stigma, rights and advocacy, trauma and disasters). An online training platform will facilitate exchange among trainers, PSWs and staff members of different study sites. The platform will provide training and supervision, and help build an international PSW community.

UPSIDES peer support will be provided without hierarchy and judgement, taking into account that mental health states may be variable. UPSIDES PSWs will focus on: providing tangible supports; role modelling that recovery is possible; sharing experiences of mental health and ill-health; and promoting hope, a sense of control and opportunities. Specific tasks include: initial assessment of strengths and resources to build on; practical support with daily life as needed (e.g. accompany to appointments or activities); support during crises; and actively promote recovery planning. Providing an additional recovery group setting is possible and recommended. UPSIDES peer support will be delivered for up to six months, with a minimum of three contacts. Weekly or biweekly meetings are recommended, but frequency may vary, depending on the needs of service users, PSWs, and study sites. The intervention manual provides additional materials to be used by the PSWs during the intervention. To support the implementation process, organisational-readiness workshops are held across trial sites. Participants allocated to the control group (wait list) will start receiving the intervention after completion of follow-up (month 12).

Control

The control intervention is treatment-as-usual as provided at each of the respective UPSIDES-RCT the study sites:

Ulm: Psychiatric routine care in Germany is mainly provided by psychiatric hospitals, psychiatric outpatient clinics and office-based psychiatrists and psychotherapists. In addition, a broad spectrum of nonmedical vocational, residential and psychosocial services are provided by vocational rehabilitation centres, community mental health care centres and different types of residential facilities. The Department of Psychiatry and Psychotherapy II at Ulm University is responsible for the provision of mental health care in a large catchment area in rural Bavaria (North and Middle Swabia, population 
671,000). Multidisciplinary teams (psychiatrists, psychologists, social workers, nurses, occupational therapists) offer the full range of pharmacological and psychosocial interventions in the large inpatient unit, two-day care units, an outpatient clinic, and a home treatment team (mobile crisis intervention). The Department collaborates closely with office-based psychiatrists and psychotherapists in the area.

Hamburg: For psychiatric routine care see Ulm above. The University Medical Centre Hamburg-Eppendorf (UKE) is one of the largest hospitals in the City of Hamburg. The Department of Psychiatry and Psychotherapy has multidisciplinary teams who provide inpatient, outpatient and outreach (Crisis Resolution Teams, Assertive Community Treatment) mental health services in a large catchment area of several districts in Hamburg and cooperates closely with various service providers in the region.

Butabika: Psychiatric services in Kampala are provided in the form of outpatient clinics at general hospitals as well as inpatient and outpatient care at the National Referral Hospital at Butabika. Physical health care, psychotherapies as well as social interventions and reintegration are provided at Butabika before service users are discharged back to their homes. Rehabilitation is provided at the Occupational Therapy department at Butabika, as there are no public community-based public mental health rehabilitation facilities. Treatment as usual will therefore comprise psychopharmacological as well as regular psychosocial care and occupational therapy provided in hospital on an inpatient or outpatient basis at Butabika Hospital

Dar es Salaam: Mental health services in Tanzania are decentralised, starting from primary care facilities which often serve as an entry point for all kinds of service users. At the district hospitals psychiatric nurses do triaging, referring and refilling medication for people with mental illness considered stable. People with severe and complicated mental illness are referred to either tertiary or psychiatric hospitals for specialised care. At a tertiary hospital (Department of Psychiatry and Mental Health at Muhimbili National Hospital, Dar es Salaam), inpatient and outpatient mental health services are provided. Providers include psychiatrists, occupational therapists, social workers, psychiatric nurses and clinical psychologists. Tanzania experiences a considerable shortage of psychiatrists, mostly of psychiatrists working at tertiary or psychiatric hospitals. Mental health services provided include psychotherapy, psychosocial rehabilitation, vocational skills training, family intervention, cognitive enhancement therapy and psychoeducation.

Beer Sheva: Mental health care in Israel is provided by psychiatric hospitals, psychiatric outpatient clinics and office-based psychiatrists and psychotherapists. Psychiatric rehabilitation services are provided through the mandatory rehabilitation basket law and include a wide range of services in the community including vocational, residential and psychosocial services and programs and community mental health care centres. The Yozma Derech Halev program specializes in supporting consumers who wish to work in rehabilitation or clinical services. It is independent of other services and supports consumers in numerous organisations who are employed in multidisciplinary mental health teams.

Pune: Mental health care in India is broadly delivered through public and private mental health facilities with in-patient and out-patient departments, with limited community-based services. However, these are 
based on a medical model of care. Services at public mental health facilities are provided at a nominal cost and are usually overburdened and under-resourced. Mental health care is often not available, accessible, acceptable nor of good quality, leading to a care gap of $70-92 \%$ for persons with mental illness. The Hospital for Mental Health in Ahmedabad, Gujarat, caters to the city of Ahmedabad (8 million population approximately), with an in-patient facility of 300 clients, and outpatient unit of 150 clients every day. Peer support volunteers are financially supported by the State at this site. Treatment as usual will therefore comprise psychopharmacological as well as regular psychosocial care and occupational therapy provided in hospital on an inpatient and outpatient basis at the hospital.

\section{Outcomes}

Primary outcome is social inclusion at $\mathrm{t}_{2}$ ( 8 months). This time point has been chosen because it is shortly after the end of the intervention but also gives enough time for changes in social inclusion to take place. Social inclusion is a key outcome in global mental health [29] and will be measured with the Social Inclusion Scale (SIS [30]) which is a service-user-reported measure with 16 items answered on a 4-point Likert scale ("not at all"; "not particularly"; "yes a bit"; "yes definitely"), yielding a total score consisting of the prorated sum over all 16 . The total score can range from 16-64 with higher scores representing higher levels of social inclusion. The SIS has previously shown adequate psychometric properties [30] which will be further established for the different language versions to be used in this trial. Secondary outcomes are empowerment (Empowerment Scale, ES [31]), hope (HOPE scale [32]), recovery (Stages of Recovery, STORI-30 [33]), and health and social functioning (Health of the Nations Outcome Scales, HoNOS [34]). Established state-of-the-art translation guidelines [35] will be followed to translate and locally validate the standardised measures (including administration instructions) used, with most attention paid to the psychometric evaluation of the primary outcome. See Puschner et al. [13] for further details on translation.

Participant timeline

Figure 3 gives an overview of this trial's participant timeline for the major stages enrolment, allocation, and assessments in line with SPIRIT recommendations ("SPIRIT figure").

Recruitment

Recruitment of study participants will vary among sites due to their differences in mental health service systems. Potential participants will be approached in various ways, including outpatient/ community mental health services, patient and carer organisations, local newspapers, social media, community leaders, and word-of-mouth. Duration of recruitment period is 12 months, starting January 2020.

\section{Allocation}

Participants will be randomly assigned to either control or intervention group with a 1:1 allocation as per a computer-generated randomisation schedule stratified by site using permuted blocks of random sizes. 
The block sizes will not be disclosed, to ensure concealment. Participants will be randomised by an independent unit (Institute for Medical Biometry and Epidemiology, UUlm, Germany) using the software ROM [36]. All participants who give consent for participation and fulfil the inclusion criteria will be randomised. Allocation concealment will be ensured, as the service will not release the randomisation code until the participant has been recruited into the trial and the baseline assessments completed.

Randomisation will be requested by the staff member responsible for recruitment and clinical interviews from UUIm's Institute for Medical Biometry and Epidemiology by e-mail. The randomisation form includes site ID, participant ID, gender of participant, date of informed consent and approval of eligibility. The requesting research worker will get a response by mail within one working day. For participants in the intervention group, the research worker will inform the local PSW team and the participant. Throughout the study, the randomisation will be conducted by UUlm's Institute for Medical Biometry and Epidemiology in order to keep the data management and the statistician blind to the study allocation for as long as the data bank is open. The randomisation list remains with UUIm's Institute for Medical Biometry and Epidemiology for the duration of the study. Thus, randomisation will be conducted without any influence of the principal investigators or raters.

Blinding

Due to the nature of the intervention, neither participants nor peer support workers can be blinded. Efforts will be made to blind staff collecting data during study visits, e.g. by separating recruitment tasks from data collection, as feasible. Researchers analysing study data will be blinded until the entire analysis has been completed, as described above.

Trial management and oversight

The Trial Management Group (TMG) consists of leads of study sites and is responsible for identification, recruitment and follow-up of study participants, data collection, and adherence to the study protocol. Oversight bodies Trial Steering Committee (TSC) and Data Safety and Monitoring Board (DSMB) are independent from the sponsor and will be continuously informed of study progress including data quality issues. The three members of the TSC will ensure that the trial is conducted in line with Good Clinical Practice. The TSC will have ultimate responsibility for the trial and will assume primacy over the DSMB and TMG. The TSC can prematurely terminate the trials, e.g. in case of violations of patient safety. The DSMB will safeguard the interests of the study participants and monitor the data collected in the trial.

Statistical analysis and power calculation

Analyses will start once baseline data have been collected and cleaned. Descriptives of all outcome measures will be produced, and outcome trajectories from $t_{0}$ to $t_{3}$ will be examined via exploratory analyses. The intervention's effect on primary and secondary outcomes will be tested by means of random-effect regression models including a fixed group effect over time, allowing the inclusion of cases 
with incomplete (unbalanced) data across panels [37]. Post-hoc analyses will examine early (at $t_{1}$ ) and late effects $\left(a t t_{3}\right)$ for primary and secondary outcomes.

Sample size calculation was performed for testing whether the primary outcome (social inclusion) at $t_{2}$ is affected by allocation. For six study sites, three time points, and estimated panel attrition of $10 \%$ at each time point, $\mathrm{N}=558$ participants ( $\mathrm{N}=93$ per site) are needed to detect a small effect size ( $0.25 \mathrm{SD}$ units) with a power of 0.80 at a two-tailed significance level of 0.05 . Sample size calculation was done using the RMASS program (www.rmass.org) for a three-level mixed-effects linear regression model for the analysis of longitudinal data, assuming a linear effect over time, compound symmetry for error variance covariance, a person-level covariance (Int, Cov, Slope) of 0.300, 0.150, 0.100, and a centre-level covariance (Int, Cov, Slope) of 0.050, 0.025, 0.020.

Cost-Effectiveness Analysis

Measures

Comprehensive societal costs of mental illness will be estimated for each participating country using an adapted version of the Client Sociodemographic and Service Receipt Inventory [38] at t0-t3. The CSSRIUPSIDES will be adapted for use at all sites in order to assess mental health service use and productivity losses due to mental illness. If not already available, country-specific unit costs of health and social care services will be determined by investigating publicly accessible sources (price lists, catalogues of fees and charges), by expert interviews or by calculations of staff and capital costs for used services drawing on the principles from the reference case for global health costing, recently developed by the Global Health Cost Consortium. All costs will be converted into international \$ using purchasing power parities (PPP) [39]. Quality-adjusted life years (QALYs) will be estimated using the EQ-5D 3L at t0-t3 which is used in most multinational cost-utility studies [40] and available in the languages of all study sites. As countryspecific population-based value sets are not available for most study sites, QALYs will be estimated on the basis of the European EQ-5D visual analogue scale (VAS) for all countries [41].

Data analysis

Incremental cost-utility ratios (ICUR) will be estimated for each country as the ratio between the average difference in total costs of illness and the average QALY difference over 12 months. Stochastic uncertainty of incremental cost-effectiveness ratios will be assessed by nonparametric bootstrapping with 2.000 replications. The maximum willingness-to-pay (MWTP) necessary to cover $95 \%$ of the ICER variance will be estimated by means of the cost-effectiveness acceptability curves [42]. In order to take into account the economic differences between participating countries, country-specific MWTP thresholds suggested by Woods et al. [43] and Leech et al. [44] will be applied.

Net monetary benefit regression models with random effects for the UPSIDES treatment, fixed country effects and fixed treatment by country interaction effects will be computed to estimate the impact of country specific differences on cost-utility [45]. Individual net-monetary benefit values will be computed 
for each study participant by multiplying the individual country specific QALYs with MWTP thresholds ranging from 0 to $50,000 \$$ (steps will be defined on the basis of results from the incremental cost-utility analysis (ICUA) and subtracting country specific individual costs [45]. Marginal effects of the net monetary benefit regression models will be computed to estimate country-specific net-monetary benefits for defined MWTP thresholds. Robust standard errors will be estimated to account for skew distribution of net-benefit values $[46,47]$. Results from the primary data ICUA will be used to develop a decision tree model [48] to simulate cost-utility and health budget impact of the UPSIDES intervention for each country over a five year time frame [49]. The sensitivity analysis will consider variance of program coverage and equity of service access [50].

Participants in the process evaluation include service users, peer support workers, mental health workers and other key informants involved in the UPSIDES intervention. Peer support workers and service users will be recruited from the participants in the RCT (see above). Purposive sampling strategies will be applied for mental health workers and key informants. Mental health workers will be recruited from multidisciplinary teams who cooperate with peer support workers. Key informants will be local stakeholders with relevant expertise relating to implementation of peer support work, including clinicians and managers who employ peer support workers.

Quantitative part of the process evaluation

Information on important process variables will be collected from different stakeholders (Table 1). Data analysis will apply random-effect regression models and structural equation modelling including process variables as moderators or mediators of effect. Additional multivariate analyses will be carried out to analyse the effect of fidelity including dose (number, frequency and duration of peer support sessions, recorded by routinely administered M\&E forms) and implementation outcomes. 
Table 1

UPSIDES RCT process measures

\begin{tabular}{|c|c|c|c|c|c|c|c|}
\hline Variable & Scale & \# of & Raters & & \multicolumn{3}{|c|}{ Timing $^{\mathrm{a}}$} \\
\hline \multicolumn{5}{|c|}{ Measures as part of RCT data assessments } & $t_{0}$ & $t_{1}$ & $t_{2}$ \\
\hline $\begin{array}{l}\text { Experiences of peer } \\
\text { support }\end{array}$ & Brief INSPIRE [51] & 5 & SU & $279 / 47$ & & $x$ & $x$ \\
\hline Fidelity & $\begin{array}{l}\text { UPSIDES peer support } \\
\text { fidelity scale [59] }\end{array}$ & $\begin{array}{l}32 \\
25\end{array}$ & $\begin{array}{l}\text { SU } \\
\text { PSW }\end{array}$ & $\begin{array}{l}279 / 47 \\
60 / 10\end{array}$ & & $x$ & \\
\hline \multicolumn{5}{|l|}{ Other measures ${ }^{a}$} & $\mathrm{~T}_{0}$ & $\mathrm{~T}_{1}$ & $\mathrm{~T}_{2}$ \\
\hline $\begin{array}{l}\text { Motivations, } \\
\text { competencies and } \\
\text { relationship } \\
\text { characteristics }\end{array}$ & $\begin{array}{l}\text { Recovery-oriented peer } \\
\text { provider work-role model } \\
\text { and prototype measure } \\
\text { (ROPP) [60] }\end{array}$ & 29 & PSW & $60 / 10$ & $x$ & $x$ & $\mathrm{x}$ \\
\hline Empowerment & $\begin{array}{l}\text { Empowerment Scale (ES) } \\
\text { [31] }\end{array}$ & 28 & PSW & $60 / 10$ & $\mathrm{x}$ & $x$ & $\mathrm{x}$ \\
\hline Recovery & $\begin{array}{l}\text { Stages of Recovery } \\
\text { (STORI-30) [33] }\end{array}$ & 30 & PSW & $60 / 10$ & $x$ & $x$ & $\mathrm{x}$ \\
\hline \multirow[t]{2}{*}{$\begin{array}{l}\text { Recovery } \\
\text { orientation }\end{array}$} & \multirow[t]{2}{*}{$\begin{array}{l}\text { Recovery Self-Assessment } \\
\text { (RSA) [61] }\end{array}$} & 36 & $\begin{array}{l}\text { Mental } \\
\text { health } \\
\text { workers }\end{array}$ & $30 / 5$ & \multicolumn{2}{|l|}{$x$} & $x$ \\
\hline & & 36 & $\begin{array}{l}\text { Key } \\
\text { informants }\end{array}$ & $12 / 2$ & \multicolumn{2}{|l|}{$x$} & $x$ \\
\hline \multicolumn{8}{|c|}{ 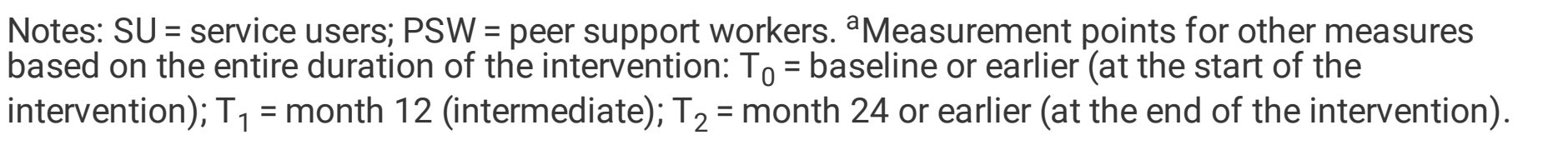 } \\
\hline
\end{tabular}

Qualitative part of the process evaluation

In this part of the study the goal is to gain a deep multi-layered contextual understanding about the impact and experiences of the UPSIDES peer support intervention among stakeholders. To this purpose, the qualitative part of the process evaluation comprises four different qualitative studies. These studies will differ in terms of objectives according to the specific target groups, and will address: (1) Service users' experiences of the peer support intervention; (2) Peer support workers' occupational development; (3) Practitioners' experiences of peer support; and (4) Key informants' experiences of peer support. 
A common overall methodology shared by each of these studies is described below and followed by detailed information on each of the individual studies. Table 2 gives an overview of qualitative studies which are part of the process evaluation.

Table 2

Overview of qualitative studies

\begin{tabular}{|c|c|c|c|c|c|}
\hline \multirow[t]{2}{*}{ \# } & \multirow[t]{2}{*}{ Participants } & \multirow[t]{2}{*}{ Method } & \multirow[t]{2}{*}{ Sample size per site } & \multicolumn{2}{|c|}{ Time point } \\
\hline & & & & $\mathrm{T}_{0}$ & $\mathbf{T}_{2}$ \\
\hline 1 & service users & interviews & $6-8$ & & $x$ \\
\hline 2 & peer support workers & focus groups & 2 groups $(3-7)$ & & $\mathrm{x}$ \\
\hline 3 & mental health workers & focus groups & 1 group $(6-8)$ & $x$ & $x$ \\
\hline 4 & key informants & focus groups & $4-6$ & $\mathrm{x}$ & $\mathrm{x}$ \\
\hline \multicolumn{6}{|c|}{$\begin{array}{l}\text { Notes: Measurement points for other measures based on the entire duration of the intervention: } T_{0}= \\
\text { baseline or earlier (at the start of the intervention); } T_{2}=\text { month } 24 \text { or earlier (at the end of the } \\
\text { intervention). }\end{array}$} \\
\hline
\end{tabular}

\section{Overall methodology}

Each study will follow these steps: (i) Provision of verbal and written introduction to the study (participant information) and obtaining written consent (consent form). (ii) Conduct and electronically record interviews or focus groups according to flexible topic guides. (iii) Collection of sociodemographic data. (iv) Verbatim transcription and pseudonymisation of audio recording. (v) Translation of field notes and transcripts into English. (vi) Thematic analysis by grouping and structuring of relevant themes that answer key issues as per study objectives.

Data analysis will involve reviewing all field notes, reading and re-reading all the transcripts for familiarization, consensual coding and generating themes. The data analysis will be conducted by a core group, to be supported by partners at each study site involved in data collection as needed. Data analysis will adhere to the following steps: (i) Based on 1-2 interviews with participants, the task lead will develop preliminary codes and categories (themes). (ii) Preliminary codes and categories (themes) will be reviewed by the core group and commented upon. (iii) Modifications of the coding tree will be commented upon by the core group. The final coding tree will be discussed and revised as necessary until consensus is reached. (iv) Subsequent inclusion of interviews and modifications of sub-categories (themes). (v) Reviewing, refining and defining themes. (vi) Validation of the coding structure with each local site.

A code book will be developed using the data from all sites, then will be shared across sites for review, discussion and standardization. The pre-determined code book will be used to develop notes, data 
managed into units of information that cover broad categories with grouping of relevant emerging themes of importance. Each site will comment on the emerged themes, and the core analysis group will finalise the themes.

The study-specific core group will decide on a software to be used for data management and text retrieval (e. g. QSR International's NVivo 12 qualitative software, MAXQDA). The focus will be on the semantic content of the data (as responses to semi-structured questions). Quality will be improved by the use of multiple analysts to ensure that a range of perspectives inform the interpretation of the data; the use of verbatim quotes for each theme, to ensure the interpretation is as close to the data as possible; and local validation to maximise cross-cultural validity of the coding framework.

\#1: Service users' experiences of peer support intervention

Objectives: In order to identify factors that contribute to the impact of peer support on service users, this study will explore service users' views on positive/ negative effects of peer support, and factors which moderate the positive/negative effects of peer support on social inclusion, (self)stigmatization, empowerment, hope, recovery, and illness concepts. By using an open approach, the study will shed light on service users' everyday experiences of the intervention beyond what is already known or expected regarding the mechanisms of impact including active ingredients and conditions for optimal experiences as well as barriers and contextual influences.

Participants: Following a mixed-method approach, 6-8 service users from the intervention group at each site will be purposively selected at the end of intervention using their quantitative outcome data to include those with and without benefit (1:1) of peer support with regard to social inclusion and experiences of peer support. Participants will be selected based on their ratings on the Social Inclusion Scale (SIS [30]) and Brief INSPIRE [51] and grouped into "low responders" (with a combination of low scores on the SIS and low scores on Brief INSPIRE) and "high responders" (with a combination of high scores on the SIS and high scores on Brief INSPIRE).

Methods: Since an open approach allows for a deeper insight into personal meaning systems of the interviewee [52], semi-structured interviews will be conducted at the end of intervention to capture service users' experiences with and subjective effects of peer support, and their attitudes towards peer support. Interviewers will use a flexible interview guide including 6-8 open questions to capture everyday experiences with peer support (e.g. talks, activities), subjective appraisal of the positive/negative effects of peer support (e.g. self-esteem, self-stigma, knowledge) and attitudes towards provision of peer support within mental health settings (e.g. contextual factors, barriers and facilitators).

\#2: Peer support workers' occupational development

Objective: To evaluate the impact of training and implementation on PSWs' occupational development, to identify PSWs' views on what contributes to or hinders successful implementation of peer support, and 
PSWs' attitudes towards the benefits and challenges of the intervention. The study will address PSWs' gender-specific views on their occupational roles, facilitators and barriers, and resources and needs.

Participants: Two focus groups (one male, one female) per site with 3-7 participants in each of the six sites, for 12 focus groups total.

Methods: To allow for comparison as well as to capture the diversity of PSW characteristics and activities across countries, focus group guidelines will include two parts: (1) Questions for PSWs across all sites focusing on (a) PSWs' experiences and perspectives in their trained roles including training and working as PSWs in this project; (b) PSWs' views on personal benefit and challenges when using lived experiences in their role as a PSW; (c) PSWs' views regarding effects on service users; and (d) barriers, facilitators and needs for successful implementation of PSW. (2) Context-sensitive questions depending on specific country stage of implementation and cultural characteristics focusing on obstacles and challenges of the PSW intervention at study sites. At study sites where the peer support model is new (e.g. Ulm, Dar es Salaam), questions will focus on inner and outer setting factors relevant to the implementation of peer support, e.g. local values and norms, power dynamics, organizational facilitators and barriers to implement peer support and to develop PSWs occupational roles. At study sites with more advanced implementation (e.g. Beer Sheva, Butabika), questions will focus on PSWs' experiences in assimilation and sustainability of their role, benefits and challenges with the use of lived experiences (e.g. selfdisclosure, boundaries), and organisational facilitators and barriers to enhancing and sustaining peer support and to developing PSWs occupational roles (e.g. financial arrangements).

\section{\#3: Practitioners' experiences of peer support}

Objective: To investigate (a) the impact on the multidisciplinary team of PSWs joining and working in the team ("impact on team culture"); (b) the impact on the individual clinician of working alongside a PSW as a colleague ("impact on clinician"); (c) implementation issues that helped or hindered the successful implementation of PSW in the team ("implementation"); and (d) the impact perceived by the clinician on service users who received peer support ("impact on service user").

Participants: Mental health workers working in the same team as an UPSIDES PSW.

Methods: Between 6 and 8 mental health workers in each site will take part in a focus group before and after the intervention. A replacement clinician will be identified for follow-up if a baseline focus group attendee is no longer available (e.g. because they have moved jobs). Participant diversity in age, gender and years since qualifying will be maximised. Data will be collected using an open focus group guide based on an implementation scale developed on a systematic review, pertaining to elements of organisational culture, training, role clarity, resourcing, and access to a peer network and more that came up as crucial for successful peer support implementation [16]. In addition, at baseline and follow-up, focus group participants will be asked to fill-out a 16 item questionnaire (Mental Illness: Clinicians' Attitudes Scale, MICA [53]) in order to assess changes of stigmatising attitudes towards people with mental illness following working with PSWs. 
\# 4: Key informants' experiences of peer support

Objectives: To explore the practical consequences on dealing with the promises and challenges of peer support from the perspectives of key informants and to assess implementation outcomes in line with the Consolidated Framework for Implementation Research (CFIR [15]). This focus group study will evaluate stakeholders' views on (a) perceived changes/difficulties prior to the intervention vs. implementation outcome; (b) barriers and facilitators for successful implementation of peer support in the given institution, and (c) the need for changes or alterations to make the intervention work effectively in a specific context.

Participants: Key informants will be defined as experts with specific authorities and/or responsibilities in regard to the implementation processes at study sites. Experts are supposed to have specific contextual (organisational) knowledge about implementation of peer support in mental health settings (e.g. policy makers, representatives of service user, provider, and funder organisations). Experts will be selected according to the local structures of mental health services.

Methods: At each site, focus groups with 4-6 key informants will be held at the beginning and end of the intervention. Purposive sampling strategies will be applied based on study site researchers' knowledge about local relevant key informants. Topics covered will include acceptability, appropriateness and feasibility, to be guided by the CFIR Interview Guide Tool [54], including characteristics at the levels of the intervention (e.g. advantages, risks/costs, cultural adaptability), the outer setting (e.g. barriers and facilitators for meeting service users' needs, policies and incentives), the individual (e.g. knowledge, values), and the intervention processes (e.g. obstacles and facilitators during planning, execution, and evaluation).

\section{Discussion}

With a balanced representation from high-, middle- and low-income countries, at different stages of peer support, UPSIDES represents a significant contribution to international implementation research on peer support. The UPSIDES-RCT offers an opportunity to explore the essential components necessary to create a peer support model in mental health care, while providing the evidence required to sustain and eventually scale-up the intervention. UPSIDES involves an effort to internationally implement and evaluate a peer support intervention for people with severe mental illness across low-middle and highincome countries, harmonising core elements across different cultural and organisational dimensions. Given the scarcity of mental health workers in LMICs and recent paradigm shift towards mental health recovery in high-income countries, involvement of peers in mental health care has the potential to improve and to transform mental health services in low-, middle- and high-resource settings.

This study addresses several knowledge gaps and limitations in previous RCT designs and is in line with calls to continue to research and improve the quality of studies on peer support in mental health $[6,55]$. First, evaluating the development of an effective peer intervention using a longitudinal design combining both quantitative and qualitative methods has been previously recommended [56]. Second, the qualitative 
component of the study will yield insights into the complex processual aspects of implementing peer support; for example, what is the role of a PSW and what do peer providers do to successfully create change, both for recipients and for organizations? Third, the RCT addresses ethical concerns regarding the use of comparison groups in intervention studies for vulnerable populations, by adopting a wait-list design. Finally, as much as possible this study protocol follows best practices for the minimization of research biases, while taking into account differences in local contexts [57].

Previous reviews of randomised trials of peer support have highlighted the heterogeneity of interventions and outcome measures used in many studies, positing that lack of standardisation may contribute to null or mixed results in this area of research (e.g. [6]). The current study addresses these concerns by developing and testing a peer support intervention based on core elements common to all participating study sites, and further investigates processes of change that have previously been identified as relevant to mental health peer support interventions. Further, it will focus on social inclusion as the primary outcome, alongside other outcomes directly related to personal recovery, in line with established definitions of mental health peer support $[8,56,58]$. In addition, the study will include mental health and economic outcomes reported to be missing from many studies of peer support, according to previous reviews $[6,55]$.

Conducting an international implementation research study will allow for the identification of universal versus local/contextual elements of the intervention, contributing to the evidence base for peer support and its theoretical underpinnings. It will characterise what this intervention looks like in different high-, middle- and low-income countries, and specify contextual factors required for its successful implementation. Identifying non-modifiable and modifiable components of peer support will help to inform the implementation of peer support in other countries and settings.

\section{Limitations}

A multicentre RCT can prove challenging, particularly in low resource settings and given differences in mental health care structures. For example, aligning timelines for tasks can be challenging with varied infrastructural and contextual challenges. Ethical review protocols and committee recommendations vary across settings, requiring flexibility within the overall framework without compromising on quality and fidelity to the model. While overall there has been much involvement in the development of the intervention by users and peer support workers, the study planning was more heavily reliant upon researchers in academic institutions. It is also necessary to plan for the high likelihood of attrition among peer staff, who may need to take sick-leave or drop out unexpectedly. Proper supports and accommodations for PSWs will be put in place to maximise retention and sustainability.

\section{Conclusions}

UPSIDES is the first multisite RCT to study peer support in a range of low-, middle- and high-income countries, addressing a number of geographical, methodological and other knowledge gaps in international research on mental health peer support. By explicitly studying process as well as outcomes, 
UPSIDES asks not just whether peer support works in these settings, but how, in order to provide practical guidance on the implementation and scale-up of peer support in different settings. Ultimately, UPSIDES aims to inform mental health policy, implementation and practice, to ensure that the perspectives and potential contributions of people with lived experience are taken into account.

\section{Abbreviations}

\begin{tabular}{|c|c|}
\hline Beer Sheva & $\begin{array}{l}\text { Department of Social Work, Ben Gurion University of the Negev, Beer Sheva, Israel } \\
\text { (study site) }\end{array}$ \\
\hline Butabika & Butabika National Referral Hospital, Uganda (study site) \\
\hline $\begin{array}{l}\text { Dar es } \\
\text { Salaam }\end{array}$ & Ifakara Health Institute, Dar es Salaam, Tanzania (study site) \\
\hline EU & European Union \\
\hline Hamburg & $\begin{array}{l}\text { Department of Psychiatry, Universitätsklinikum Hamburg-Eppendorf, Germany (study } \\
\text { site) }\end{array}$ \\
\hline HIC & High-income country \\
\hline LMIC & Low- or middle-income country \\
\hline London & $\begin{array}{l}\text { Centre for Global Mental Health, London School of Hygiene and Tropical Medicine, UK } \\
\text { (study site) }\end{array}$ \\
\hline Nottingham & Institute of Mental Health, University of Nottingham, UK (study site) \\
\hline PSW & Peer support worker \\
\hline Pune & Centre for Mental Health Law and Policy, Pune, India (study site) \\
\hline RCT & Randomised controlled trial \\
\hline SMI & Severe mental illness \\
\hline Ulm & Department of Psychiatry II, Ulm University, Germany (coordinating study site) \\
\hline UPSIDES & $\begin{array}{l}\text { Using Peer Support in Developing Empowering Mental Health Services (project } \\
\text { acronym) }\end{array}$ \\
\hline
\end{tabular}

\section{Declarations}

\section{Ethics and consent to participate}

Participants who provided valid written informed consent will be included. Each potential participant in this research project, prior to consent, will be clearly informed of the study goals, possible adverse events and the possibility to refuse to participate or to withdraw consent without any adverse consequences. Informed consent will be asked only of persons able to freely understand and question. The study 
protocol has been approved by Ulm University's Ethics Commission, Germany (ref: 254/19); Local Psychological Ethics Commission at the Centre for Psychosocial Medicine, Hamburg, Germany (ref: LPEK-0095); Indian Law Society (ref: ILS/37/2018); Human Subjects Research Committee of Ben-Gurion University, Israel (ref: 1787-1); Uganda National Council for Science and Technology (ref: SS 4990). Approval pending from Tanzania ethics committee.

\section{Consent for publication}

Not applicable.

\section{Availability of data and material}

Not applicable.

\section{Competing interests}

The author(s) declare that they have no competing interests.

\section{Funding}

UPSIDES has received funding from the European Union's Horizon 2020 research and innovation programme under grant agreement No 779263. This publication reflects only the authors' view. The Commission is not responsible for any use that may be made of the information it contains. The funding body had no role in the design of the study and in writing the manuscript.

\section{Authors' contributions}

GM and JK contributed equally to this paper (shared first authorship). BP, MS and AMS conceived the trial. RK conceived the cost-effectiveness analysis. GM, SK and BP conceived the process evaluation. BM provided statistical expertise. All authors have revised the manuscript critically for important intellectual content, and gave final approval of the version to be published.

\section{Acknowledgements}

The study Using Peer Support In Developing Empowering Mental Health Services (UPSIDES) is a multicentre collaboration between the Department for Psychiatry and Psychotherapy II at UIm University, Germany (Bernd Puschner, coordinator); the Institute of Mental Health at University of Nottingham, UK (Mike Slade); the Department of Psychiatry at Universitätsklinikum Hamburg-Eppendorf, Germany 
(Candelaria Mahlke); Butabika National Referral Hospital, Uganda (David Basangwa); the Centre for Global Mental Health at London School of Hygiene and Tropical Medicine, UK (Grace Ryan); Ifakara Health Institute, Dar es Salaam, Tanzania (Donat Shamba); the Department of Social Work at Ben Gurion University of the Negev, Beer Sheva, Israel (Galia Moran); and the Centre for Mental Health Law and Policy, Pune, India (Jasmine Kahla).

We are grateful for their valuable contributions to this work to

the UPSIDES consortium members Ramona Hiltensperger, Philip Wolf, Thomas Becker (Germany); Dean Thompson, Dave Bailie, Cerdic Hall (UK); Fileuka Ngakongwa (Tanzania); Max Lachmann, Paula GaberEpstein (Israel); Soumitra Pathare (India);

the members of the UPSIDES-RCT Trial Steering Committee (Dr Carrie Brooke-Sumner, South African Medical Research Council, Cape Town, South Africa; Dr Dickens H Akena, Makerere University College of Health Sciences, Kampala, Uganda; Prof Peter Brieger, kbo-Isar-Amper-Klinikum, Munich, Germany;

the members of the UPSIDES Data Safety and Monitoring Board (Prof Stefan Wesner, Ulm University, Ulm, Germany; Jochen Kaiser, Bezirkskliniken Schwaben, Günzburg, Germany; David Jemba, IT consultant, Entebbe, Uganda); and to

the members of the UPSIDES International Advisory Board (Marianne Farkas, Boston University, USA; Michelle Funk, World Health Organization, Geneva, Switzerland; Nigel Henderson, Penumbra, Edinburgh, Scotland; Sylvia Kaaya, Muhimbili University of Health and Allied Sciences, Dar es Salaam, Tanzania; Stefan Weinmann, Charite Medical University, Berlin, Germany; and Charlene Sunkel, Global Mental Health Peer Network, Johannesburg, South Africa).

\section{Trial Status}

- Protocol version number and date: UPSIDES RCT study protocol, Version 4.1, consented by all contributors on 08 Mai 2019.

- Date recruitment began: Recruitment has not yet begun. First participant to be included in January 2020

- Approximate date when recruitment will be completed: December 2020.

\section{References}

1. Repper J, Perkins R. Social inclusion and recovery: a model for mental health practice. Kent: Baillière Tindall; 2003.

2. Puschner B. Peer support and global mental health. Epidemiol Psychiatr Sci. 2018;27:413-4. doi:10.1017/S204579601800015X. 
3. Solomon P. Peer support/peer provided services underlying processes, benefits, and critical ingredients. Psychiatr Rehabil J. 2004;27:392. doi:10.2975/27.2004.392.401.

4. Simpson EL, House AO. Involving users in the delivery and evaluation of mental health services: systematic review. BMJ. 2002;325:1265. doi:10.1136/bmj.325.7375.1265.

5. Slade M, Amering M, Farkas M, Hamilton B, O'Hagan M, Panther G, et al. Uses and abuses of recovery: implementing recovery-oriented practices in mental health systems. World Psychiatr. 2014;13:12-20. doi:10.1002/wps.20084.

6. Pitt V, Lowe D, Hill S, Prictor M, Hetrick SE, Ryan R, Berends L. Consumer-providers of care for adult clients of statutory mental health services. Cochrane Database Syst Rev. 2013;3:CD004807. doi:10.1002/14651858.CD004807.pub2.

7. Vally $Z$, Abrahams $L$. The effectiveness of peer-delivered services in the management of mental health conditions: a meta-analysis of studies from low- and middle-income countries. Int J Adv Counselling. 2016;38:330-44. doi:10.1007/s10447-016-9275-6.

8. Davidson L, Bellamy C, Guy K, Miller R. Peer support among persons with severe mental illnesses: a review of evidence and experience. World Psychiatr. 2012;11:123-8. doi:10.1016/j.wpsyc.2012.05.009.

9. Walker G, Bryant W. Peer support in adult mental health services: A metasynthesis of qualitative findings. Psychiatr Rehabil J. 2013;36:28-34. doi:10.1037/h0094744.

10. Pathare S, Kalha J, Krishnamoorthy S. Peer support for mental illness in India: an underutilised resource. Epidemiol Psychiatr Sci. 2018:1-5. doi:10.1017/S2045796018000161.

11. Fan Y, Ma N, Ma L, Xu W, Steven Lamberti J, Caine ED. A community-based peer support service for persons with severe mental illness in China. BMC Psychiatr. 2018;18:170. doi:10.1186/s12888-0181763-2.

12. Vandewalle J, Debyser B, Beeckman D, Vandecasteele T, van Hecke A, Verhaeghe S. Peer workers' perceptions and experiences of barriers to implementation of peer worker roles in mental health services: A literature review. Int J Nurs Stud. 2016;60:234-50. doi:10.1016/j.ijnurstu.2016.04.018.

13. Puschner B, Repper J, Mahlke C, Nixdorf R, Basangwa D, Nakku J, et al. Using Peer Support in Developing Empowering Mental Health Services (UPSIDES): Background, rationale and methodology. Ann Glob Health. 2019;85:1-10. doi:10.5334/aogh.2435.

14. Gillard S, Gibson SL, Holley J, Lucock M. Developing a change model for peer worker interventions in mental health services: a qualitative research study. Epidemiol Psychiatr Sci. 2015;24:435-45. doi:10.1017/S2045796014000407.

15. Damschroder LJ, Aron DC, Keith RE, Kirsh SR, Alexander JA, Lowery JC. Fostering implementation of health services research findings into practice: a consolidated framework for advancing implementation science. Implementation Sci. 2009;4:50. doi:10.1186/1748-5908-4-50.

16. Ibrahim N, Thompson D, Nixdorf R, Kalha J, Mpango R, Moran G, et al. A systematic review of influences on implementation of peer support work for adults with mental health problems. Soc Psychiatr Psychiatr Epidemiol 2019, online first. doi:10.1007/s00127-019-01739-1. 
17. Chan A-W, Tetzlaff JM, Gøtzsche PC, Altman DG, Mann H, Berlin JA, et al. SPIRIT 2013 explanation and elaboration: guidance for protocols of clinical trials. BMJ. 2013;346:e7586. doi:10.1136/bmj.e7586.

18. Rosen A, Strathdee G, Slade M, Powell R. Threshold Assessment Grid (TAG): The development of a valid and brief scale to assess the severity of mental illness. Soc Psychiatr Psychiatr Epidemiol. 2000;35:78-85. doi:10.1007/s001270050011.

19. Stratford AC, Halpin M, Phillips K, Skerritt F, Beales A, Cheng V, et al. The growth of peer support: an international charter. J Ment Health. 2017:1. doi:10.1080/09638237.2017.1340593.

20. Repper J, Aldridge B, Gilfoyle S, Gillard S, Perkins R, Rennison J. Peer support workers: theory and practice (ImROC Briefing Paper 5). 2013. http://www.imroc.org/wp-content/uploads/5ImROC-PeerSupport-Workers-Theory-and-Practice.pdf. Accessed 21 Mar 2017.

21. Utschakowski J, Sielaff G, Bock T, Winter A, editors. Experten aus Erfahrung: Peerarbeit in der Psychiatrie [Experts from experience: peer work in psychiatry]. Cologne: Psychiatrie Verlag; 2016.

22. The Brain Gain Project. Peer Support Worker Training Manual: Developed by the Butabika East London Link with the support of the Ugandan Ministry of Health.; 2018.

23. World Health Organization. Creating peer support groups in mental health and related areas: WHO QualityRights training to act, unite and empower for mental health (pilot version). Geneva.

24. Pathare S, Kalha J, Joseph T, Funk M, Drew-Bold N, Khenti A. QualityRights Gujarat. In: Kerrigan P, Bhattacharya S, Peel S, Sá MR, Kishore R, Wade A, editors. Mental health: pasts, current trends and futures. York, UK: Orient Blackswan; 2017. p. 64-78.

25. Healthy Options Project Tanzania. Lay counsellors guide and manual (pre-tested draft 1.0). Dar es Salaam, Tanzania; 2015.

26. Peer2Peer. Vocational training course: PEER2PEER Project Partners; 2015.

27. Moran GS. The consumer movement and peer providers in Israel. Epidemiol Psychiatr Sci. 2018;27:420-6.

28. Mahlke Cl, Nixdorf R, Repper J, UPSIDES study group. UPSIDES Peer Support Training Manual and Workbook. UPSIDES Study; 2019.

29. Baumgartner JN, Burns JK. Measuring social inclusion-a key outcome in global mental health. Int J Epidemiol. 2014;43:354-64. doi:10.1093/ije/dyt224.

30. Secker J, Hacking S, Kent L, Shenton J, Spandler H. Development of a measure of social inclusion for arts and mental health project participants. J Ment Health. 2009;18:65-72. doi:10.1080/09638230701677803.

31. Rogers ES, Ralph RO, Salzer MS. Validating the Empowerment Scale with a multisite sample of consumers of mental health services. Psychiatr Serv. 2010;61:933-6. doi:10.1176/ps.2010.61.9.933.

32. Snyder CR, Harris C, Anderson JR, Holleran SA, al e. The will and the ways: Development and validation of an individual-differences measure of hope. J Pers Soc Psychol. 1991;60:570-85. doi:10.1037//0022-3514.60.4.570. 
33. Andresen R, Caputi P, Oades L. Development of a short measure of psychological recovery in serious mental illness: The STORI-30. Australas Psychiatr. 2013;21:267-70.

doi:10.1177/1039856213476352.

34. Wing JK, Beevor AS, Curtis RH, Park SB, Hadden S, Burns A. Health of the Nation Outcome Scales (HoNOS). Research and development. Br J Psychiatry. 1998;172:11-8. doi:10.1192/bjp.172.1.11.

35. Wild D, Eremenco S, Mear I, Martin M, Houchin C, Gawlicki M, et al. Multinational trialsrecommendations on the translations required, approaches to using the same language in different countries, and the approaches to support pooling the data. The ISPOR Patient-Reported Outcomes Translation and Linguistic Validation Good Research Practices Task Force Report. Value Health. 2009;12:430-40. doi:10.1111/j.1524-4733.2008.00471.x.

36. Rohlmann F, Muche R, Goldschmidt L. Randomisierung in klinischen Studien: praktische Umsetzung mit dem Randomisationsprogramm ROM [Randomisation in clinical trials: practical implementation with the randomisation software ROM]. In: Schweizer B, Großmann C, Meule M, Gaus W, editors. Dokumentation - der Schritt ins3. Jahrtausend - 8. DVMD-Tagung in Ulm. Ulm: Universitätsverlag Ulm; 2004. p. 168-171.

37. Raudenbush SW, Bryk AS. Hierarchical linear models: Applications and data analysis methods. 2nd ed. Thousand Oaks, Calif.: Sage Publ; 2010.

38. Chisholm D, Knapp M, Knudsen HC. Client Socio-Demographic and Service Receipt Inventory European Version: Development of an instrument for international research: EPSILON Study 5 . $\mathrm{Br} \mathrm{J}$ Psychiatry. 2000;177:28s-33. doi:10.1192/bjp.177.39.s28.

39. OECD Data. Conversion rates - Purchasing power parities (PPP). 2019. https://data.oecd.org/conversion/purchasing-power-parities-ppp.htm. Accessed 17 Oct 2019.

40. Oppong R, Jowett S, Roberts TE. Economic evaluation alongside multinational studies: a systematic review of empirical studies. PLoS ONE. 2015;10:e0131949. doi:10.1371/journal.pone.0131949.

41. Greiner W, Weijnen T, Nieuwenhuizen M, Oppe S, Badia X, Busschbach J, et al. A single European currency for EQ-5D health states. Results from a six-country study. Eur J Health Econ. 2003;4:22231. doi:10.1007/s10198-003-0182-5.

42. Willan AR, Briggs AH. Statistical analysis of cost-effectiveness data. Chichester: Wiley; 2006.

43. Woods B, Revill P, Sculpher M, Claxton K. Country-level cost-effectiveness thresholds: initial estimates and the need for further research. Value Health. 2016;19:929-35. doi:10.1016/j.jval.2016.02.017.

44. Leech AA, Kim DD, Cohen JT, Neumann PJ. Use and misuse of cost-effectiveness analysis thresholds in low- and middle-income countries: trends in cost-per-DALY studies. Value Health. 2018;21:759-61. doi:10.1016/j.jval.2017.12.016.

45. Hoch JS, Briggs AH, Willan AR. Something old, something new, something borrowed, something blue: A framework for the marriage of health econometrics and cost-effectiveness analysis. Health Econ. 2002;11:415-30. doi:10.1002/hec.678.

46. Huber PJ, Ronchetti E. Robust statistics. 2nd ed. Hoboken: Wiley; 2009. 
47. White H. A heteroskedasticity-consistent covariance matrix estimator and a direct test for heteroskedasticity. Econometrica. 1980;48:817. doi:10.2307/1912934.

48. Briggs AH, Claxton K, Sculpher M. Decision modelling for health economic evaluation. Oxford: Oxford University Press; 2006.

49. Sullivan SD, Mauskopf JA, Augustovski F, Jaime Caro J, Lee KM, Minchin M, et al. Budget impact analysis-principles of good practice: report of the ISPOR 2012 Budget Impact Analysis Good Practice II Task Force. Value Health. 2014;17:5-14. doi:10.1016/j.jval.2013.08.2291.

50. Asaria M, Griffin S, Cookson R. Distributional cost-effectiveness analysis: a tutorial. Med Decis Making. 2016;36:8-19. doi:10.1177/0272989X15583266.

51. Williams J, Leamy M, Bird V, Le Boutillier C, Norton S, Pesola F, Slade M. Development and evaluation of the INSPIRE measure of staff support for personal recovery. Soc Psychiatr Psychiatr Epidemiol. 2015;50:777-86. doi:10.1007/s00127-014-0983-0.

52. Curry LA, Nembhard IM, Bradley EH. Qualitative and mixed methods provide unique contributions to outcomes research. Circulation. 2009;119:1442-52. doi:10.1161/CIRCULATIONAHA.107.742775.

53. Gabbidon J, Clement S, van Nieuwenhuizen A, Kassam A, Brohan E, Norman I, Thornicroft G. Mental Illness: Clinicians' Attitudes (MICA) scale-psychometric properties of a version for healthcare students and professionals. Psychiatr Res. 2013;206:81-7. doi:10.1016/j.psychres.2012.09.028.

54. CFIR Research Team. CFIR Interview Guide Tool. 2014. http://cfirwiki.net/guide/app/index.html\#/. Accessed 17 Oct 2019.

55. Gillard S. Peer support in mental health services: where is the research taking us, and do we want to go there? J Ment Health. 2019;28:341-4. doi:10.1080/09638237.2019.1608935.

56. Mahlke Cl, Krämer UM, Becker T, Bock T. Peer support in mental health services. Curr Opin Psychiatr. 2014;27:276-81. doi:10.1097/YC0.0000000000000074.

57. Mancini MA. Strategic storytelling: an exploration of the professional practices of mental health peer providers. Qual Health Res. 2019;29:1266-76. doi:10.1177/1049732318821689.

58. Gillard S, Foster R, Gibson S, Goldsmith L, Marks J, White S. Describing a principles-based approach to developing and evaluating peer worker roles as peer support moves into mainstream mental health services. Mental Health and Social Inclusion. 2017;21:133-43. doi:10.1108/MHSI-03-20170016.

59. Hiltensperger R, Mahlke Cl, Nakku J, Mpango R, Ryan G, Nixdorf R, et al. UPSIDES peer support fidelity scale: UPSIDES Study; 2019.

60. Moran GS. A recovery-oriented peer provider (ROPP) work-role model and prototype measure. Am J Psychiatr Rehabil. 2018;20:346-68. doi:10.1080/15487768.2017.1374895.

61. O'Connell M, Tondora J, Croog G, Evans A, Davidson L. From rhetoric to routine: assessing perceptions of recovery-oriented practices in a state mental health and addiction system. Psychiatr Rehabil J. 2005;28:378-86. doi:10.2975/28.2005.378.386. 


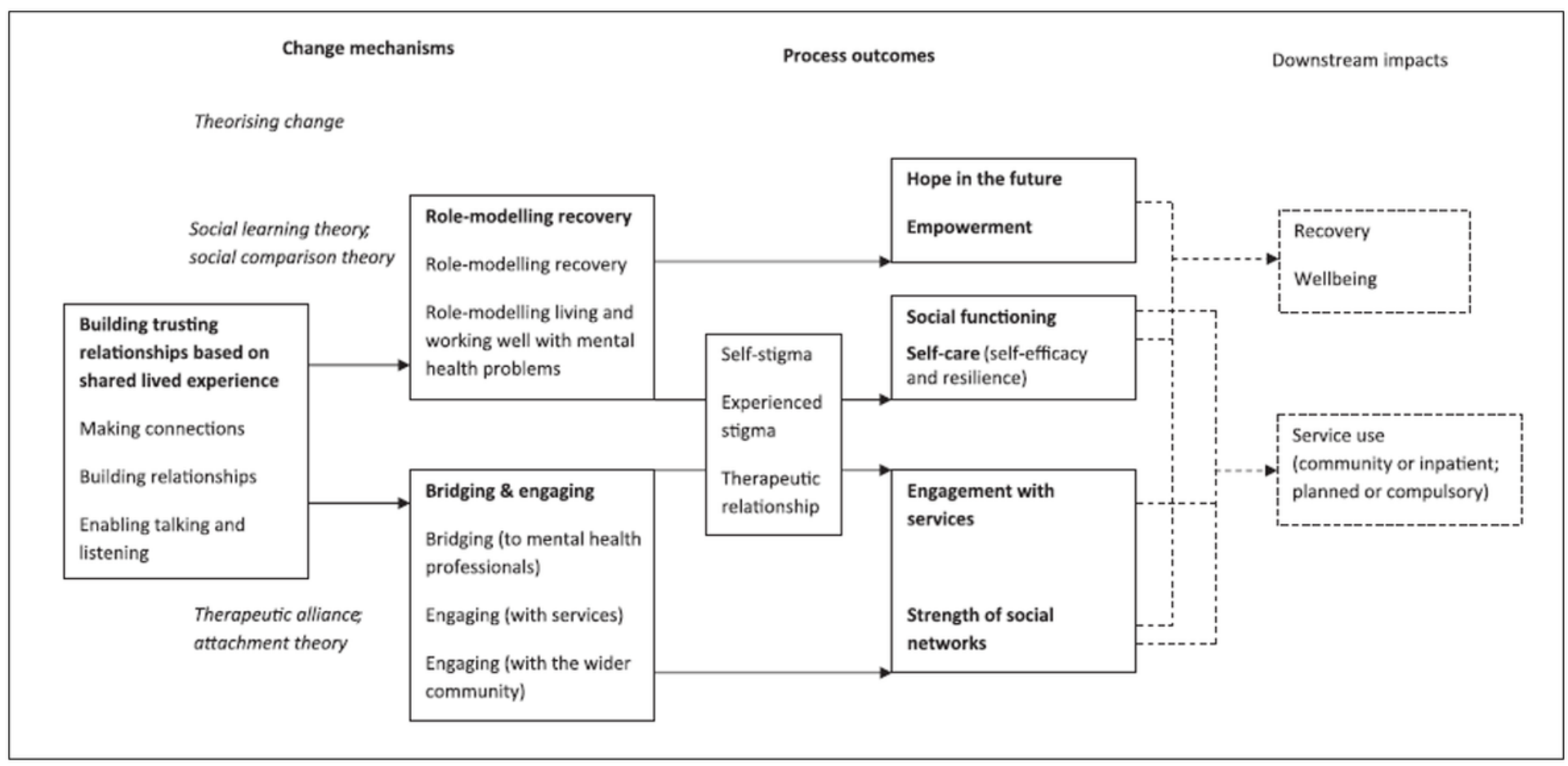

\section{Figure 1}

Change model underpinning peer worker interventions [14].

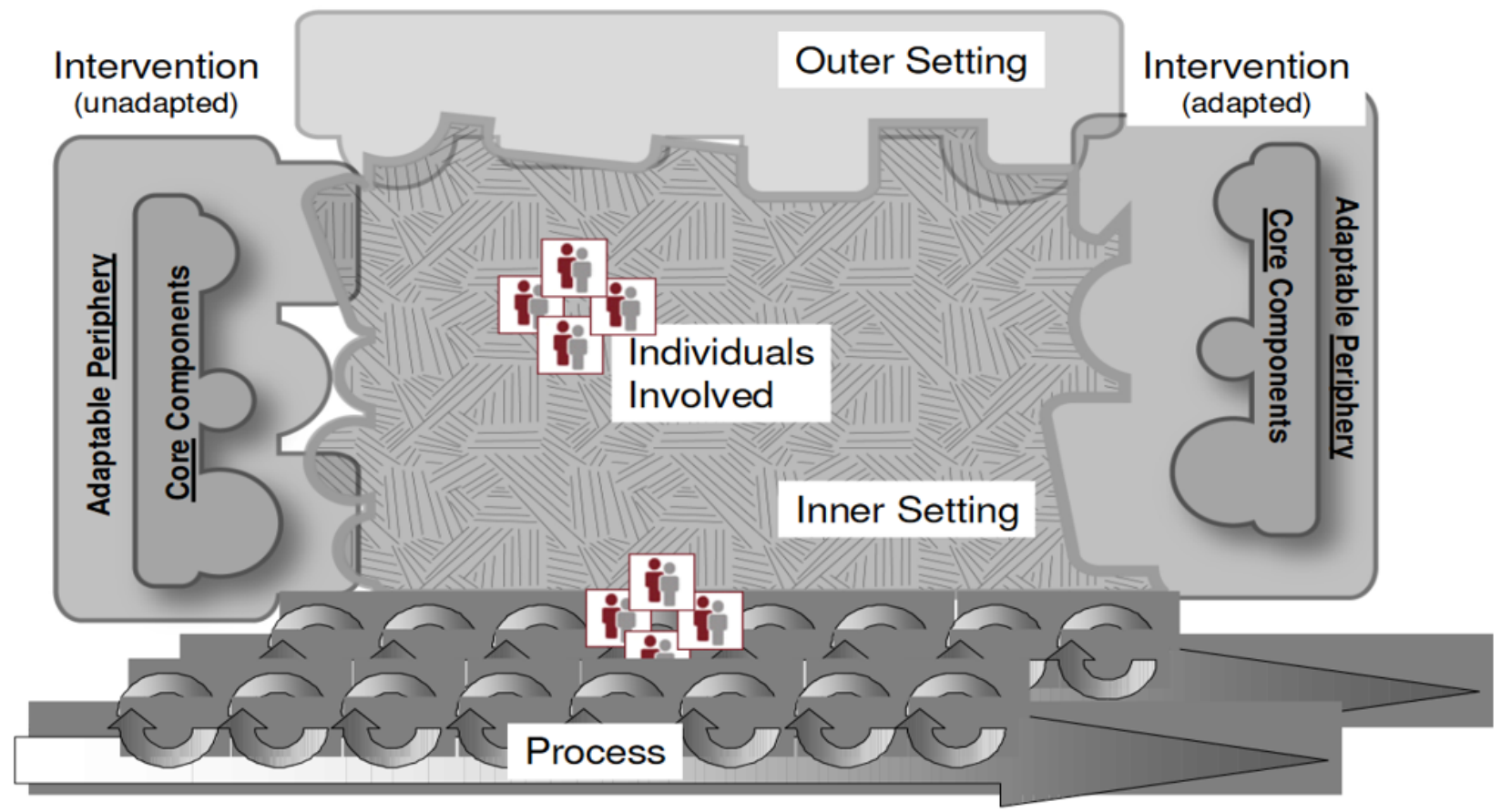

Figure 2 
Change model underpinning peer worker interventions [14].

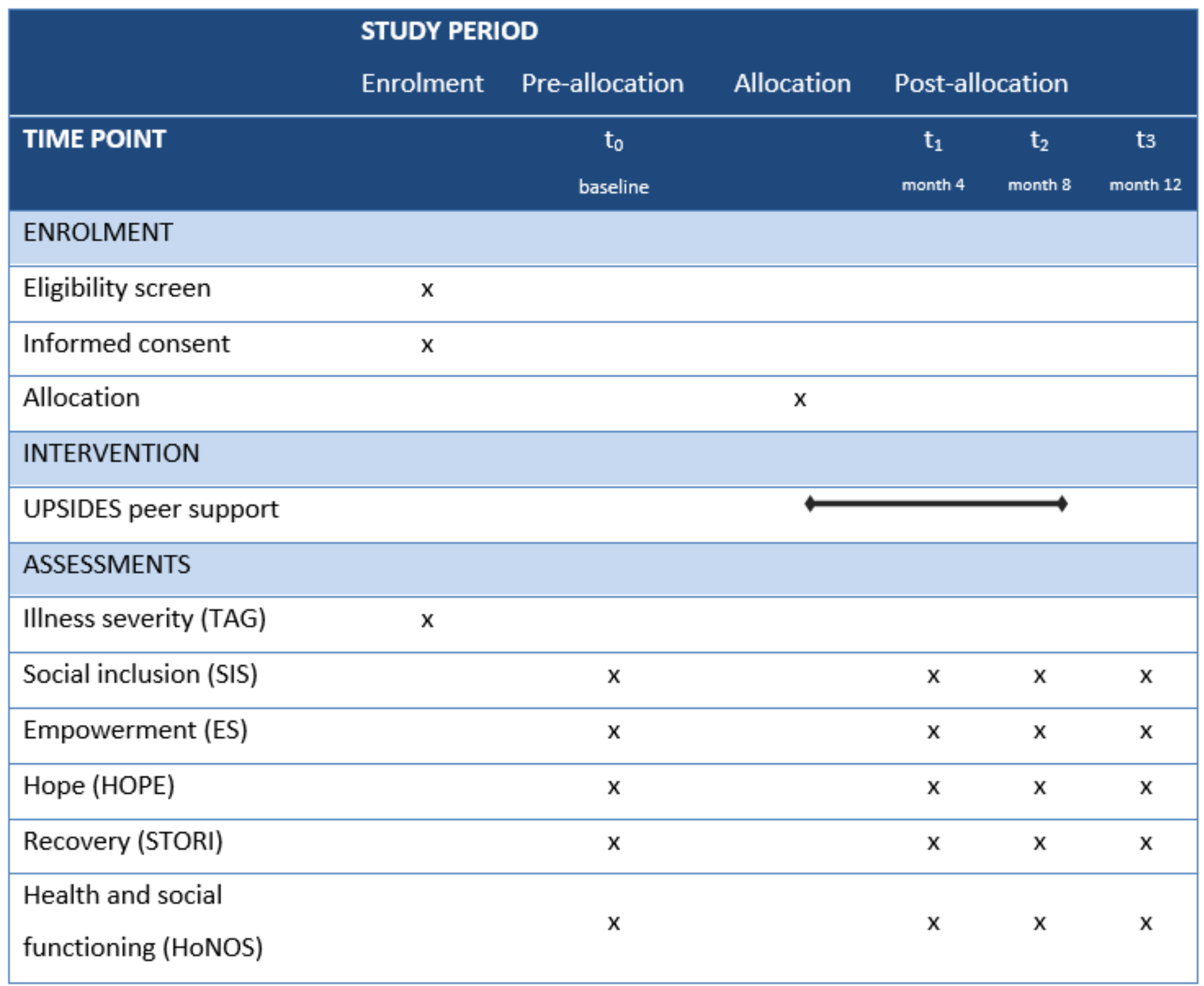

\section{Figure 3}

UPSIDES RCT participant timeline ("SPIRIT figure").

\section{Supplementary Files}

This is a list of supplementary files associated with this preprint. Click to download.

- SPIRITChecklistUPSIDESRCT.pdf 